\title{
Repercusion Ocupacional de las Amputaciones Traumaticas en Dedos de la Mano por Accidente de Trabajo
}

\section{Impact Occupational of Traumatic Amtutation In Fingers of the Hand by Work Accident}

\author{
Lía Clara López Sullaez \\ Especialista en Medicina del Trabajo, \\ Caja de Salud de Caminos y RA, La Paz - Bolivia \\ René Estrada Ruíz \\ Médico residente - Ortopedia y Traumatología, \\ Hospital Obrero No 1, La Paz - Bolivia \\ Correspondencia: \\ Dra. Lia Clara López Sullaez \\ Médico del Trabajo \\ CAJA DE SALUD DE CAMINOS Y RA \\ Zona Amor de Dios calle $1 n^{\circ} 106$ \\ La Paz - Bolivia \\ Tfno: 73039562 \\ E-mail: claralopez47@hotmail.es \\ E-mail: lopezliaclara@latinmail.com
}

Resumen

Introducción: Las amputaciones de los dedos de la mano incapacitan al trabajador de forma permanente para realizar sus actividades de la vida diaria y ocupacional, dependiendo de los dedos afectados.

Objetivo: Determinar la repercusión ocupacional de las amputaciones traumáticas de los dedos de la mano por accidente de trabajo según los dictámenes de invalidez.

Materiales y métodos: Estudio descriptivo retrospectivo, que se realizó mediante 112 formularios de dictamen de invalidez emitidos por la Entidad Encargada de Calificar de las gestiones 2005 a 2008.

Resultados: La ocupación fue recortada en el 42,9\% (3/7) de los trabajadores con amputación del pulgar y en el 72,4\% (21/29) cuando la amputación fue del índice.

La amputación de 2 y 3 dedos hizo que la ocupación fuera recortada en 64\% (16/25) y 66,7\% (2/3) respectivamente. La ocupación fue adaptada en la amputación de 4 dedos (4/4) y transmetacarpiana (2/2); y confinada cuando afectó más de 5 dedos $(2 / 2)$.

Conclusión:La amputación de los dedos de la mano produce diferentes grados de minusvalía ocupacional de acuerdo al dedo o dedos lesionados, por lo que se deben implementar medidas de prevención en las diferentes empresas para disminuir la accidentabilidad.

(Med Segur Trab (Internet) 2009; 55 (217): 41-48)

Palabras clave: amputación traumática, discapacidad, falanges de los dedos de la mano. 


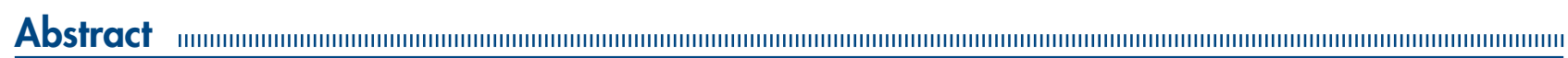

Introduction: Amputations of the fingers disabling worker for a permanent form fir to realize their activities of daily living and occupational, depending on the affected fingers.

Aim: To determine the impact of occupational traumatic amputations of the fingers by work accident according to disability form.

Materials and methods: Retrospective descriptive study, realized trhought 112 forms of disability issued by the Entidad Encargada de Calificar, during years 2005 to 2008.

Results: The occupation was clip in $42.9 \%$ (3/7) of workers with amputation of the thumb and $72.4 \%$ $(21 / 29)$ when it was in the forefinger.

Amputation of two and three fingers was clipped occupation in 64\% (16/25) and 66.7\% (2/3) respectively. The occupation was adapted when the amputation was of four fingers (4/4) and transmetacarpiana (2/2); and confined when affected more than five fingers $(2 / 2)$.

Conclusion: Amputation of the finger produces different grades of occupational disability according to injured finger or fingers, reason by they must implement preventive measures in different companies for reduce injuries.

(Med Segur Trab (Internet) 2009; 55 (217): 41-48)

Key words: amputation traumatic, occupational disability, finger phalanges. 


\section{INTRODUCCIÓN}

Las manos son el principal instrumento para la manipulación física del medio siendo el pulpejo la zona con más terminaciones nerviosas del cuerpo humano y fuente de información táctil sobre el entorno ${ }^{1,2}$.

El principal uso de las manos es el de tomar y sostener objetos, aunque de estos derivan muchos más por la gran versatilidad y precisión de movimientos que posee, siendo usadas como "utensilios" para comer, en el lenguaje de señas, la escritura y para aliviar el dolor mediante técnicas de masaje denominándose "tacto estructurado"”.

Según la Administración de Salud, Higiene y Seguridad Ocupacional Norteamericana (O.S.H.A), de los 2 millones de trabajadores norteamericanos incapacitados cada año, alrededor de 400.000 presentan lesiones de las manos, siendo la localización mas frecuente en los dedos $(72 \%)^{1}$. Los costos de las lesiones de mano en México, genera un total de 2'525.086 días de incapacidad temporal, con un costo promedio por día de 66.7 dólares, generando una erogación en subsidios de 168.473.737 dólares ${ }^{2,4}$. El sector industrial registra que el $60 \%$ de las amputaciones es en la mano, siendo los obreros que trabajan con metales los que más amputaciones traumáticas registra $(6,7 \%)^{4}$. En nuestro país existen pocos estudios acerca de la epidemiología de los accidentes de trabajo, entre los cuales se encontró uno realizado en empresas textiles, en el que reporta que del total de accidentes de trabajo el $54,8 \%$ corresponde a mano, de los cuales el $2,4 \%$ es por amputación traumática 5 .

Las lesiones de la mano adquieren gran importancia, por ser una región anatómica de excepcional valor, por su utilización en casi todas las profesiones u ocupaciones. Cualquier nivel de amputación, lleva a un grado de incapacidad que puede limitar al individuo incluso para realizar actividades tan elementales como la alimentación y el aseo personal, de manera permanente, requiriendo posteriormente la readaptación laboral o cambio de puesto de trabajo ${ }^{4}$.

El presente estudio pretende determinar cual es la repercusión ocupacional de las amputaciones traumáticas en dedos de la mano por accidente de trabajo debido a que no existen datos acerca de esta problemática y posteriormente implementar medidas de prevención, mejorando de ésta manera los sistemas de seguridad industrial en las empresas.

\section{MATERIALES Y MÉTODOS}

Estudio descriptivo retrospectivo, que se realizó mediante el uso de datos secundarios individuales (Formulario único de Dictamen de invalidez emitido por la Entidad Encargada de Calificar el grado de Invalidez) de las gestiones 2005 a 2008, obteniéndose 112 formularios de trabajadores con amputación traumática en dedos de la mano por accidente de trabajo, se excluyeron del estudio los accidentes en itinere o trayecto, formularios incompletos y en los que se calificaba otra patología aparte de la amputación. Los datos fueron recogidos en un cuestionario diseñado para el estudio, por personal capacitado.

La variable independiente fue el nivel de amputación traumática en dedos de la mano, las variables dependientes minusvalía ocupacional, patrón funcional de la mano perdido y las variables de control: edad, sexo, ocupación, rubro de empresa, tiempo de experiencia.

Para medir la minusvalía ocupacional se utilizó el Manual de normas de evaluación y calificación del grado de invalidez de Bolivia ${ }^{6}$, el que clasifica las ocupaciones en ocho grupos que son: habitualmente ocupado (desempeño en la misma ocupación), desocupado intermitente (desempeño en la misma ocupación pero debe suspender ocasionalmente por 1 o 2 días sus actividades), ocupación recortada (desempeño en la misma ocupación pero con dificultades para realizar algunas tareas), ocupación adaptada 
(no puede volver a desempeñarse en su ocupación anterior, pero puede realizar otra ocupación normalmente), ocupación reducida (la persona ha tenido que limitar su actividad sólo a media jornada), ocupación restringida (no puede realizar su ocupación habitual u otra si no tiene algunas condiciones especiales), ocupación confinada (solo realiza actividades en la casa y por periodos cortos), sin ocupación (no realiza ninguna actividad).

Los datos obtenidos fueron procesados mediante el paquete estadístico SPSS 11.5.

\section{RESULTADOS.}

La edad promedio de los trabajadores con amputación traumática en dedos de la mano por riesgo profesional fue 33,2 años +/-10,1 ( IC $_{95 \%} 31,4-35,1$ años), con una edad mínima de 17 años y la máxima de 60 años. Por grupo etario se encontró que el 72,3\% (81 casos) tenía entre 21 a 40 años, el 23,2\% (26 casos) entre 41 a 60 años y el 4,46\% (5 casos) fue menor a 20 años. El 90,2\% (101 casos) correspondió al sexo masculino y el 9,8\% (11 casos) al femenino.

Las empresas en las que ocurrieron las amputaciones traumáticas correspondieron al rubro de servicios (alcaldía, empresa municipal de aseo, servicio de caminos) en $21,4 \%$ ( 24 casos), madereras 18,8\% (21 casos), minería en 11,6\% (13 casos), alimentos 10,8\% (12 casos), construcción y afines 5,4\% (6 casos), textil 4,5\% (5 casos), plásticos 3,6\% (4 casos), avícola $1,8 \%$ ( 2 casos) y otros $22,4 \%$ ( 25 casos).

La ocupación de los trabajadores fue ayudantes en el 24,1\% (27 casos), operadores en $22,3 \%$ ( 25 casos), mecánicos en $8,1 \%$ ( 9 casos) y mineros $7,2 \%$ (8 casos). Tabla 1.

\begin{tabular}{lcc} 
Tabla 1 - Ocupación de los trabajadores con amputaciones traumáticas en dedos \\
\cline { 2 - 4 } Ocupación & Frecuencia & Porcentaje \\
\hline Ayudante & 27 & 24.1 \\
Operador & 25 & 22.3 \\
Mecánico & 9 & 8.1 \\
Minero & 8 & 7.2 \\
Militar & 7 & 6.3 \\
Obrero & 7 & 6.3 \\
Empleado & 4 & 3.6 \\
Encargado Sección & 4 & 3.6 \\
Limpieza & 5 & 4.5 \\
Electricista & 3 & 2.7 \\
Carpintero & 4 & 3.6 \\
Portero & 2 & 1.8 \\
Otros & 7 & 6.3 \\
Total & 112 & 100 \\
\hline
\end{tabular}

Fuente: Elaboración propia, Entidad Encargada de Calificar, 2005-2008.

El tiempo de experiencia promedio fue 52,6 +/- 67 meses ( IC $_{95 \%}$ 40,1 - 65,1 meses), el mínimo tiempo de experiencia fue de 0,03 meses ( 8 días) y el máximo 320 meses (26 años y 8 meses), al realizar la estratificación de esta variable se encontró que el 26,8\% (30 casos) tenía experiencia menor a 6 meses, 8,9\% (10 casos) 6 a 12 meses, 25\% (28 
casos) de 13 a 36 meses, 6,3\% (7 casos) 37 a 60 meses y 33\% (37 casos) tenía experiencia mayor a 60 meses ( 5 años).

La mano izquierda fue afectada en 50\% (56 casos), la mano derecha en 48,2\% (54 casos) y ambas manos en 1,8\% ( 2 casos). Los dedos que más se afectaron fueron el índice en $25,9 \%$ ( 29 casos), el medio en 17\% (19 casos), el anular en 10,7\% (12 casos). Tabla 2.

Tabla 2 - Frecuencia de amputaciones traumáticas por dedo afectado

\begin{tabular}{lcc}
\hline Dedos Afectados & Frecuencia & Porcentaje \\
\hline Índice & 29 & 25.9 \\
2 Dedos & 25 & 22.3 \\
Medio & 19 & 17.0 \\
Anular & 12 & 10.7 \\
Meñique & 9 & 8.0 \\
Pulgar & 7 & 6.3 \\
4 Dedos & 4 & 3.6 \\
3 Dedos & 3 & 2.7 \\
Transmetarcarpiana & 2 & 1.8 \\
Más De 5 Dedos & 2 & 1.8 \\
Total & 112 & 100 \\
\hline
\end{tabular}

Fuente: Elaboración propia, Entidad Encargada de Calificar, 2005-2008.

El nivel de amputación fue interfalángica distal en 42,9\% (48 casos), interfalángica proximal 27,7\% (31 casos), metacarpofalángica 18,8\% (21 casos) e interfalágica (primer dedo) en $5,4 \%$ (6 casos). Tabla 3.

Tabla 3 - Frecuencia de amputaciones traumáticas en dedos de la mano por nivel de lesión

\begin{tabular}{lcc}
\hline Nivel De Lesión & Frecuencia & Porcentaje \\
\hline Ifd & 48 & 42.9 \\
Ifp & 31 & 27.7 \\
Mcf & 21 & 18.8 \\
If & 6 & 5.4 \\
Pulpejo & 3 & 2.7 \\
Transmetacarpiana & 2 & 1.8 \\
Carpometacarpiana & 1 & 0.9 \\
Total & 112 & 100 \\
\hline
\end{tabular}

IFD: Interfalángica distal, IFP: interfalángica proximal; IF: interfalángica; MCF: metacarpofaláncica.

Fuente: Elaboración propia, Entidad Encargada de Calificar, 2005-2008.

Los patrones funcionales (movimientos) de la mano perdidos por la amputación traumática fueron pinza fina en $27,7 \%$ (31 casos), pinza gruesa en 18,8\% (21 casos), las dos pinzas en $15,2 \%$ y todos los movimientos en 8,9\% (10 casos). Tabla 4. 
Tabla 4 - Frecuencia de patrón funcional perdido o afectado por amputaciones traumáticas en dedos de la mano

\begin{tabular}{lcc}
\hline Patrón Funcional & Frecuencia & Porcentaje \\
\hline Prensión Pulgar-Dedo O Pinza Fina & 31 & 27.7 \\
Perdida Parcial De Cilindro (Anular O Meñique Afectados) & 21 & 18.8 \\
Prensión En 3 Puntos O Pinza Gruesa & 21 & 18.8 \\
Pinzas & 17 & 15.2 \\
Todos Los Movimientos & 10 & 8.9 \\
Puño, Pinza Gruesa, Prensión & 7 & 6.3 \\
Puño & 3 & 2.7 \\
Prensión & 1 & 0.9 \\
Pinza Gruesa, Puño, Prensión, Cilindro & 1 & 0.9 \\
Total & 112 & 100 \\
\hline
\end{tabular}

Fuente: Elaboración propia, Entidad Encargada de Calificar, 2005-2008.

El desempeño ocupacional en la amputación a nivel del pulpejo e interfalángica distal fue habitualmente ocupado en $100 \%(3 / 3)$ y $60,4 \%$ (29/48) respectivamente; la ocupación fue recortada en el $64,5 \%(20 / 31)$ para amputación a nivel de interfalángica proximal, en $50 \%(3 / 6)$ para la interfalángica (pulgar) y en $33,3 \%(7 / 21)$ para la metacarpofalángica, la ocupación fue adaptada en $100 \%$ (2/2) cuando la amputación fue transmetacarpiana y $100 \%(1 / 1)$ para la carpometacarpiana. Tabla 5.

Tabla 5 - Desempeño ocupacional de acuerdo al nivel de amputación

\begin{tabular}{|c|c|c|c|c|c|c|}
\hline \multirow{2}{*}{ Nivel De Lesión } & \multicolumn{6}{|c|}{ Desempeño Ocupacional } \\
\hline & & $\begin{array}{c}\text { Habitualmente } \\
\text { Ocupado }\end{array}$ & $\begin{array}{l}\text { Ocupación } \\
\text { Recortada }\end{array}$ & $\begin{array}{c}\text { Ocupación } \\
\text { Adaptada }\end{array}$ & $\begin{array}{l}\text { Ocupación } \\
\text { Confinada }\end{array}$ & Total \\
\hline \multirow{2}{*}{ Pulpejo } & $\mathrm{N}^{\circ}$ & 3 & 0 & 0 & 0 & 3 \\
\hline & $\%$ & 100 & 0 & 0 & 0 & 100 \\
\hline \multirow{2}{*}{ Ifd } & $\mathrm{N}^{\circ}$ & 29 & 18 & 1 & 0 & 48 \\
\hline & $\%$ & 60.4 & 37.5 & 2.1 & 0 & 100 \\
\hline \multirow{2}{*}{ Ifp } & $\mathrm{N}^{\circ}$ & 8 & 20 & 3 & 0 & 31 \\
\hline & $\%$ & 25.8 & 64.5 & 9.7 & 0 & 100 \\
\hline \multirow{2}{*}{ If } & $\mathrm{N}^{\circ}$ & 2 & 3 & 1 & 0 & 6 \\
\hline & $\%$ & 33.3 & 50 & 16.7 & 0 & 100 \\
\hline \multirow{2}{*}{ Mcf } & $\mathrm{N}^{\circ}$ & 6 & 7 & 6 & 2 & 21 \\
\hline & $\%$ & 28.6 & 33.3 & 28.6 & 9.5 & 100 \\
\hline \multirow{2}{*}{ Transmetacarpiana } & $\mathrm{N}^{\circ}$ & 0 & 0 & 2 & 0 & 2 \\
\hline & $\%$ & 0 & 0 & 100 & 0 & 100 \\
\hline \multirow{2}{*}{ Carpometacarpiana } & $\mathrm{N}^{\circ}$ & 0 & 0 & 1 & 0 & 1 \\
\hline & $\%$ & 0 & 0 & 100 & 0 & 100 \\
\hline \multirow{2}{*}{ Total } & $\mathrm{N}^{\circ}$ & 48 & 48 & 14 & 2 & 112 \\
\hline & $\%$ & 42.9 & 42.9 & 12.5 & 1.8 & 100 \\
\hline
\end{tabular}

Ifd: Interfalángica Distal, Ifp: Interfalángica Proximal; If: Interfalángica; Mcf: Metacarpofaláncica. 
En la amputación del pulgar y el índice la ocupación fue recortada en 42,9\% (3/7) y $72,4 \%(21 / 29)$ respectivamente. La ocupación fue recortada en $64 \%(16 / 25)$ cuando la amputación afectó 2 dedos y en 66,7\% (2/3) cuando afectó 3 dedos; fue adaptada en 100\% (4/4) en la amputación de 4 dedos y en la amputación de más de 5 dedos la ocupación fue confinada en $100 \%(2 / 2)$. Tabla 6.

Tabla 6 - Desempeño ocupacional de acuerdo a dedo lesionado

\begin{tabular}{|c|c|c|c|c|c|c|}
\hline \multirow{2}{*}{ Dedo Lesionado } & \multicolumn{6}{|c|}{ Desempeño Ocupacional } \\
\hline & & $\begin{array}{c}\text { Habitualmente } \\
\text { Ocupado }\end{array}$ & $\begin{array}{l}\text { Ocupación } \\
\text { Recortada }\end{array}$ & $\begin{array}{l}\text { Ocupación } \\
\text { Adaptada }\end{array}$ & $\begin{array}{l}\text { Ocupación } \\
\text { Confinada }\end{array}$ & Total \\
\hline \multirow{2}{*}{ Pulgar } & $\mathrm{N}^{\circ}$ & 2 & 3 & 2 & 0 & 7 \\
\hline & $\%$ & 28.6 & 42.9 & 28.6 & 0 & 100 \\
\hline \multirow{2}{*}{ Índice } & $\mathrm{N}^{\circ}$ & 7 & 21 & 1 & 0 & 29 \\
\hline & $\%$ & 24.1 & 72.4 & 3.4 & 0 & 100 \\
\hline \multirow{2}{*}{ Medio } & $\mathrm{N}^{\circ}$ & 16 & 3 & 0 & 0 & 19 \\
\hline & $\%$ & 84.2 & 15.8 & 0 & 0 & 100 \\
\hline \multirow{2}{*}{ Anular } & $\mathrm{N}^{\circ}$ & 9 & 3 & 0 & 0 & 12 \\
\hline & $\%$ & 75 & 25 & 0 & 0 & 100 \\
\hline \multirow{2}{*}{ Meñique } & $\mathbf{N}^{\circ}$ & 8 & 1 & 0 & 0 & 9 \\
\hline & $\%$ & 88.9 & 11.1 & 0 & 0 & 100 \\
\hline \multirow{2}{*}{2 Dedos } & $\mathbf{N}^{\circ}$ & 6 & 16 & 3 & 0 & 25 \\
\hline & $\%$ & 24 & 64 & 12 & 0 & 100 \\
\hline \multirow{2}{*}{3 Dedos } & $\mathrm{N}^{\circ}$ & 0 & 1 & 2 & 0 & 3 \\
\hline & $\%$ & 0 & 33.3 & 66.7 & 0 & 100 \\
\hline \multirow{2}{*}{4 Dedos } & $\mathrm{N}^{\circ}$ & 0 & 0 & 4 & 0 & 4 \\
\hline & $\%$ & 0 & 0 & 100 & 0 & 100 \\
\hline \multirow{2}{*}{ Más De 5 Dedos } & $\mathrm{N}^{\circ}$ & 0 & 0 & 0 & 2 & 2 \\
\hline & $\%$ & 0 & 0 & 0 & 100 & 100 \\
\hline \multirow{2}{*}{ Transmetacarpiana } & $\mathrm{N}^{\circ}$ & 0 & 0 & 2 & 0 & 2 \\
\hline & $\%$ & 0 & 0 & 100 & 0 & 100 \\
\hline \multirow[b]{2}{*}{ Total } & $\mathrm{N}^{\circ}$ & 48 & 48 & 14 & 2 & 112 \\
\hline & $\%$ & 42.9 & 42.9 & 12.5 & 1.8 & 100 \\
\hline
\end{tabular}

\section{DISCUSIÓN}

Las amputaciones traumáticas de dedos de la mano son más frecuentes en el rubro de servicios, madereras y minería, siendo las ocupaciones más afectadas los ayudantes, operadores de maquinaria, mecánicos y mineros, éstos datos no se relacionan con lo que mencionan otros estudios en los que encontraron que éste tipo de lesiones ocurren con mayor frecuencia en el rubro minero ${ }^{1}$ y en la construcción ${ }^{4}$, siendo las ocupaciones más afectadas los obreros y estibadores ${ }^{4}$.

Las amputaciones traumáticas ocurren con mayor frecuencia cuando el tiempo de experiencia es menor a 6 meses y mayor a 50 meses ( 5 años), esto se explicaría en el primer caso porque el trabajador aún esta conociendo sus funciones y a veces el esta 
como eventual en la empresa y debe realizar todo tipo de funciones, además que en éste periodo puede iniciarse el empleo de maquinaria que es el principal agente de los accidentes de trabajo 5 . Los trabajadores con un tiempo de experiencia mayor a 5 años pueden sufrir accidentes debido a la confianza que sienten al cumplir sus funciones, lo que hace que a veces cometan actos inseguros, que serian la causa de las amputaciones, también algunos de éstos trabajadores producto de la edad pueden tener algunas deficiencias como auditivas, visuales que dificultarían el cumplimiento de sus actividades.

La mano que se afecta con mayor frecuencia es la izquierda y los dedos índice, medio y anular, estos datos concuerdan con los encontrados en otro estudio en el que la frecuencia de lesión del dedo índice fue $22 \%$, medio $14 \%$ y anular $14 \%$. Sin embargo la lesión de 2 dedos es menos frecuente que en nuestro estudio (22,3 versus 18,2\%) ${ }^{1,3}$. Los niveles de amputación que ocurren con más frecuencia son interfalángica distal (IFD), interfalángica proximal (IFP) y metacarpofalángica (MCF); siendo los patrones funcionales perdidos o afectados la pinza fina, gruesa y ambas pinzas que son movimientos fundamentales para desempeñar la mayor parte de las actividades de la vida diaria y ocupacional.

En relación al dedo amputado y el desempeño ocupacional se encontró que la ocupación se encuentra recortada para las amputaciones de pulgar, índice, medio y cuando se afectaron 2 dedos, esto debido a que con estos dedos se realizan los movimientos de pinza fina y gruesa que sirven para actividades tan fundamentales como la escritura o el sosten de algunos objetos pequeños. La ocupación fue confinada cuando hubo amputación de más de 5 dedos, porque el trabajador a perdido todos los movimientos de la mano que se encuentra afectada y debe prescindir de ésta mano para realizar algunas actividades. Tambien se observó que cuando la lesión es del anular o meñique los trabajadores continuan habitualmente ocupados esto posiblemente a que pueden suplantar los movimientos de éstos dedos con otros.

En conclusión la amputación de los dedos pulgar, índice y de más de dos dedos modifican el desempeño ocupacional de los trabajadores, reduciéndola o debiendo hacer algunas modificaciones para su desempeño, por esta razón se deben disminuir los accidentes de trabajo en las diferentes empresas mediante la fortificación de los sistemas de higiene y seguridad industrial, de ésta manera se disminuirá la accidentabilidad y los costos que éstos representan tanto para el empleador como para el trabajador.

\section{REFERENCIAS}

1. Sirit-Urbina Y, Fernández-D’Pool J, Lubo-Palma A. Accidentes de la mano en trabajadores de la Costa Oriental del Lago de Maracaibo del Estado Zulia, Venezuela, 1986-1993. Invest clín 2002; 43(2): 25-8.

2. Amillo S, Romero LM. Lesión laboral en mano. Formación de Seguridad Laboral. (citado de 12 enero de 2009). Disponible en la World Wide Web: http://www.borrmart.es/articulo_laboral.php?id=1925.

3. Medina CP, Pardo V. Lesiones de punta de dedos ocasionados por accidentes de trabajo, Clínica SanPedro Claver, Seguro Social. Revista Colombiana de Ortopedia y Traumatología 2001;15(1): 4-10.

4. Salinas S, Lozada ME, Rodriguez T, Fresnedo M, López P. Las lesiones por riesgo de trabajo en el instituto Mexicano del Seguro Social. Coordinación de Salud del Trabajo, Area Riesgos de Trabajo. IMSS. $1992-1996$.

5. López L, Terceros A. Características y factores contribuyentes de los accidentes de trabajo en empresas textiles gestión 2005-2006. Revista Médica 2007; 13(1): 31-8.

6. Superintendencia de pensiones, valores y seguros. Manual de normas de evaluación y calificación del grado de invalidez. La Paz - Bolivia. SPVS; 2001. 